\title{
Agile Methods Selection Model: A Grounded Theory Study
}

\author{
Mashal Kasem Alqudah ${ }^{1}$, Rozilawati Razali ${ }^{2}$, Musab Kasim Alqudah ${ }^{3}$ \\ Research Center for Software Technology and Management, FTSM \\ University Kebangsaan Malaysia Bangi, Selangor, Malaysia ${ }^{1,2}$ \\ Department of Computer Sciences, College of Computer Sciences and Engineering-Yanbu, Taibah University, Saudi Arabia ${ }^{3}$
}

\begin{abstract}
Agile methods adoption has increased in recent years because of its contribution to the success rate of project development. Nevertheless, the success rate of projects implemented using Agile methods has not completely reached its expected mark, and selecting the appropriate Agile methods is one of the reasons for such lag. Selecting the appropriate Agile methods is a challenging task because there are so many methods to select from. In addition, a lot of organizations consider the selection of Agile methods as a mammoth task. Therefore, to assist Agile team members, this study aimed to investigate how the appropriate Agile methods can be determined for different projects. Based on a Grounded Theory study, 23 Agile experts drawn from 19 teams across thirteen countries were interviewed. Hence, this study employed the Ground Theory of selecting Agile methods. Sixteen factors, grouped into five categories, have been found to affect the selection of twenty Agile methods. The nature of project (size, maturity, criticality and decomposability), development team skills (communication skills, domain knowledge, team technical skills and maturity), project constraints (cost/value/ROI, cost of change, time, scope and requirements volatility), customer involvement (collaboration, commitment and domain knowledge) and organizational culture (type of organizational culture) are the key factors that should guide Agile team members in the selection of an appropriate Agile methods based on the value these factors have for different organizations and/or different projects.
\end{abstract}

Keywords-Agile methods selection; factors; model; grounded theory analysis

\section{INTRODUCTION}

Majority of companies are inclined towards implementing effective quality systems in a timely manner through reasonable costs [1]. This is the reason of such companies shift towards Agile methods, and in this regard, it has been revealed that different factors have to be considered when selecting Agile methods [2]. The selection for different projects should be according to the nature of projects, development team proficiency, organizational culture, customer involvement and project constraints considering several alternative Agile methods, Consequently, although the Agile methods use are increasing in software development projects, there are still firms that look upon the selection of a suitable Agile method as a great insurmountable challenge. Moreover, to compound this issue, studies dedicated to the difficulties related with the Agile methods effective selection are still few and far between $[2,3]$.

Therefore, This is expected to help the members of the Agile team to select the most suitable Agile methods based on different project conditions to guarantee that that the results are successful. Accordingly, a grounded theory study was conducted to investigate how the factors that influence the selection of Agile methods can assist Agile team members in such selection.

The remaining parts of this paper is organized in the following way; in Section 2, the Agile methods background and the related techniques are discussed, and in Section 3, the research methodology used in the study to examine the selection of Agile methods is presented. This is followed by Section 4 that contains the results from the grounded theory study, and Section 5 that provides the Agile methods selection model. Lastly, in Section 6, the conclusion and future studies scope are enumerated.

\section{RELATED WORK}

In this section, the author provides a brief overview of the background of Agile methods and the major methods utilized for their selection. In this regard, a group of 17 software developers came together to work towards enhancing system development methods in 2001. They brought forward the Agile methods that have their basis on four values and twelve principles under the Agile Manifesto. Specifically, the four values lay stress on individuals and interactions, working software, customer collaboration and change response [4]. The Alliance also fine-tuned the values encapsulated in their manifesto into twelve principles in order to assist developers and project managers to understand the Agile methods and the application of their values. Some examples of the principles are listed as follows [4].

1) Face-to-face conversation is the most effective and efficient method of conveying information.

2) Welcome changing requirements at any stage of the development.

3) Self-organizing teams are very important for software development.

4) Simplicity is essential

Over the years, different Agile methods and practices have been developed to cover and achieve the above principles. For instance, different Agile methods focus on the principle of having face-to-face conversations (principle 1 above). On the other hand, XP method focuses on keeping the design of the system very simple to be able to maintain and quickly revise (principle 4 above). Kanban also limits work in progress to achieve simplicity (principle 4 above), so there is a possibility 
of changing the requirements at any stage of the development (principle 2 above), while, collective ownership is an Agile modeling practice that focuses on assisting each team member to work on any model and manage the work with other team members as a group (principle 3 above).

In the current times, several Agile methods can be used and this added to the difficulty in the selection of the most suitable one to use in specific contexts [2]. In addition, organizations find it difficult to employ the same method for all their projects implementation owing to the varying requirements of every project [5]. Also, there is no single Agile method that is capable of solving all the issues of the organization [3]. Examples of Agile methods include Kanban [6], SCRUM [7], Crystal family of methods [5] and XP [8]. Therefore, there is a necessity for discerning a way to ensure that the top suitable Agile methods are selected to suit specific projects [2]. Hence, researchers and practitioners have begun examining different selection techniques for the most appropriate Agile method to use for a specific project. Examples of the selection methods that have been proposed in literature can be found at [2]. However, examples of the current selection methods are listed as follows:

- Sayed et al. (2017) proposed a method for Agile methods selection using Analytic Hierarchy Process (AHP). Positive response in dynamic requirements, communication with the customer, incorporation of requirements changes, and the size of development team were the main factors that should be considered in the selection of Agile methods [9]. However, the selection was for Institute Examination System project and crystal family and Agile Modeling methods were the most appropriate methods for this project.

- Sharma et al. (2017) proposed a framework for Agile Development Method Selection using multi-criteria decision making. The researchers used Modified PROMETHEE with Analytic Hierarchy Process to select the appropriate method among six Agile methods (Lean Software development, DSDM, XP, Scrum, Crystal Clear and FDD [3]. Formalization and documentation, reluctance to change, practices overhead and project reliability and scalability were the main factors that should be considered during the selection. This kind of methods, by the use of matrices, require experts' knowledge of the suggested alternative to be able to select the appropriate methods [10].

\section{RESEARCH METHODOLOGY}

Prior studies in literature show the necessity to examine the Agile methods selection but as yet, no empirical findings have been reported on the demonstration of selection processes of Agile methods based on the values of an integrated selection factors. Therefore, the main objective of this study is to conduct an empirical study to investigate how the appropriate Agile methods can be determined for different projects and/or different teams in different organizations. Although the qualitative approach comprises of several research designs, there are five common qualitative research designs, and they are case study, ethnography, content analysis, grounded theory and phenomenological study. The present study used the grounded theory as the researcher found it to be the most suitable method to use to answer the research question of the research. The main reasons of conducting a GT are as follows:

1) Grounded theory is a qualitative research method, which allows the researchers to understand social interactions and behaviors. Agile methods are all about people and interactions [11].

2) Previous studies demonstrates that the selection of appropriate Agile methods was not explored in detail [3]. So, grounded theory is destined for that [11].

3) Grounded theory helps in producing theory rather than extending or verifying extant theories in what is referred to as an interesting and new prospective.

4) Grounded theory is used when examining new themes and areas and when providing a new view into an established phenomenon.

As will be explained below, a total of 23 interview have been conducted with Agile experts concerning Agile methods selection. The following sub-sections present descriptions of the GT procedures applied in this research.

\section{A. Data Collection (Interviews)}

The present study adopted semi-structured in-depth interviews with open-ended questions to help the researcher to obtain and understand the views, experiences, perceptions and viewpoints of the interviewees on Agile methods selection. Twenty-three experts were chosen from different organizations from different countries.

The 23 participants were experienced in using different Agile methods, and 20 Agile methods were noted to be used in different organizations by the participants. The methods were reported with clarification on how they have been selected.

\section{B. Data Analysis}

Grounded Theory (GT) approach was employed in data analysis. It was employed for the identification of processes and factors affecting Agile methods selection. In addition, it assists in understanding how different selection factors can be used in the selection of Agile methods. The researcher employed three coding techniques established by GT approach namely, open coding, axial coding and selective coding to analyze qualitative data [12]. Following the assignation and creation of open code, lists of open code are categorized on the basis of their codes relationship and connection. Project size, Maturity and criticality are examples of the initial codes. Axial coding process groups general categories in open coding under higher ordering headings in order to minimize the number of categories by combining similar and dissimilar into general categories. For instance, project size, maturity and criticality fall under the nature of project. Moreover, selective coding process identifies the core category that relates to the gathered data, and such core category functions as the hub for the rest of the other identified categories. For instance, that project constraints is among the main core-categories consisting of scope, time, quality and cost/value/ROI as categories, and requirements volatility and cost of change as sub-categories. 


\section{RESULT}

In the following sections the results will be reported to present Agile methods Selection theory. The results involved the analysis of the results retrieved from the interviews. That is, how the appropriate Agile methods can be determined for different projects and/or different teams in different organizations. Agile methods selection model illustrates the factors that have been found to affect the selection of an Agile methods. Each method can be used for different projects in different situations. The explanation including examples of the statements that have been obtained from the respondents are described in the next sections as follows:

\section{A. Nature of Project}

Projects are not the same in size and in criticality and as such, no single Agile method is appropriate for all organizational issues [5]. In other words, the project nature (size, maturity, criticality and Decomposability) has to be examined and kept into consideration during the selection of Agile methods.

1) Project size: The analysis revealed that project size, in many ways, influences the selection of Agile methods. With small project size (less than 10 stakeholders), XP, SCRUM, Crystal Clear, DSDM and Kanban are preferred. This is because methods such as, XP and SCRUM allow the small team to work together face-to-face when developing a project:

R13 (Project Manager): "We make a small team to do the work and we separate the tasks for them and we found DSDM and Kanban to be suitable."

R14 (Chief Knowledge Engineer): "SCRUM, Crystal Clear and XP work fine for small teams because you do not need extra collaboration and extra communication."

However, with medium project size (10 to 100 stakeholders), scaled method such as SCRUM of SCRUM, LeSS, Nexus and Crystal Red could be more appropriate:

R10 (Senior Consultant): "For big product groups, like 8 groups with eight people in each group, we can use LeSS Framework or Crystal Red. I can also use Nexus"

R8 (Director of the Agile Organisation): "SCRUM of SCRUM is directly scaled for about 20,30 or 40 stakeholders, without needing something else."

If one group of people is working on a single large project (above 100 developers), then scaling methods such as DAD, SAFe, Nexus+ and Spotify scaling methods, are appropriate as they could be used for a large group of people. Additionally, they are appropriate for large projects since there is a need for initial planning to solve dependencies issue and more architecture for the same issue, including which team understands what to implement exactly.

R10 (Senior Consultant): "I also consider the project size, if the project is large, up to 100 people, I will use Nexus and if more than 100, we follow Nexus+. This can be suitable to scale SCRUM."

R14 (Chief Knowledge Engineer): "If they are working on a single program of work, then you got to add in some project management, you got to add in work value management, you got to add cross-team collaboration, you got to add in probably some rigor tooling. Your governance processes might have to be a little bit more when you do a robust work as it will be a little bit more extreme. So, DAD, LeSS, SAFe and Spotify could guide you when the project is large."

2) Project criticality: Critical projects are those projects that might cause essential loose of money or human lives [5]. An understanding of the criticality of the project can help in determining differences in the results of applying different Agile methods. Most of the respondents mentioned that XP method and TDD Agile modeling, Lean Startup, DAD and $\mathrm{SAFe}$ are the main methods to be used in the development of critical projects:

R23: "For critical projects, you must select the process that brings the most amount of feasibility and predictability. In my opinion, the one that I've had the most successful outcomes from is from the XP point of view." Agile Coach

R5 (Project Manager): "XP practices should be adopted for critical projects and you should apply TDD."

Other respondents suggested other methods to be used for critical projects, such as Agile modeling, Lean Startup, DAD and SAFe.

R16 (Senior Consulting Partner): "If you are doing financial processing system, which is very critical, then sequence diagrams and other modeling under Agile modeling approach could be appropriate but again, if the team does not have sequence modelling skills, then this would be a bad choice."

R21 (Project Manager): "If the project is really critical, then you need to get this out of the door by UX. You need to talk about feature prioritization and what is MVP from that point of view, So, Lean Startup practices assist you in understanding how to solve critical issues."

R5 (Project Manager): "Methods like SAFe or DAD, does lead the way as to how to reduce the amount of complexity and have more flexibility within the organization and that includes reducing the criticality and the amount of dependency."

3) Project decomposability: Agile methods assist teams respond to unpredictability through incremental, iterative work cadences and empirical feedback. However, some projects are difficult to be implemented in an iterative or incremental manner. Examples of such projects could be airplane, elevator, airbus, mobile and car. They are difficult to be decomposed but they could be implemented successfully when the right approach is selected. There is a need for initial modelling, initial planning, testing regularly and milestone instead of sprints. So, Kanban, Lean Development, Lean Startup and DAD are more appropriate if the product is difficult to be decomposed:

R16 (Senior Consulting Partner): "The major components of the A380 airbus were delivered in parts even it was so difficult to decompose it. So, once again for this kind of project, I get back to initial modelling, initial planning and 
testing all the way through, which means a combination of methods is needed. However, DAD was capable of doing this and it could assist the team to release the project at a single time but build the project in pieces."

R21 (Project Manager): "If you can decompose it into sprints, you can use SCRUM, if you cannot decompose, then you can use Kanban, which allows you to use milestone."

R18 (Chief Information Officer): "Look at how they develop the rockets or Samsung S5, S6 and S7. They should make sure how the old one can still plug into the new component. So, in this case, practices of Lean approaches such as, MVP would help in understanding how such a product could be decomposed considering the customers' need."

4) Project maturity: Different product maturity at different stages of the development should be considered when selecting Agile methods. The analysis revealed that product maturity could be divided into four stages namely, legacy product, new product, development stage and maintenance stage.

If the product is a legacy product, which contains legacy code, then the appropriate methods to be used is XP method. In addition, TDD is difficult to be used in this case:

R3 (Delivery Manager): "If the team is coding legacy code, TDD will be difficult for them because the design of the code is such that some codes are really written in a way that is very difficult to test. So, TDD will always be difficult to be used for a legacy product."

R9 (Agile Adoption Change Leader): "For legacy code, XP practices such as pairing and refactoring are good at ensuring you don't create any more technical debt."

The second stage of project maturity is when the product is something new in which case, we have to start from scratch. Lean startup, TDD and DSDM are the most appropriate Agile methods for this:

R13 (Project Manager): "If I am implementing a new project, I can use DSDM but when it goes for maintenance project I will follow Kanban."

R17 (Senior Agile Developer): "We have to use TDD when there are new projects that we have no idea about it."

R16 (Senior Consulting Partner): "Typically, the Lean Startup method or we call it the exploratory life cycle, is used when you are dealing with new product development."

When the product is in the development or prototype stage, then SCRUM and/or XP would be a better choice:

R3 (Delivery Manager): "Well, as we are moving out with the prototype stage I will involve SCRUM and some XP practices."

Finally if the product is in the maintenance stage then Lean development, DevOps and Kanban methods are the appropriate methods to be used. In other words, for maintenance and support products, adopting Kanban or DevOps has higher success rates compared to SCRUM.
R11 (Product and Management Consultant): "For support and operational product blindly, a Kanban is suitable but for maintenance, Kanban \& DevOps are both suitable.”

In addition, methods such as Adaptive Software Development (ASD) and Lean development are also appropriate:

R3 (Delivery Manager): "If the customer provides clear features, and refuses to see the step by step development as the end is known through the use of product, this is where the maturity of the product comes. In this case, I will probably go with Lean Development or Kanban, because at the end of it, I'll show you the product, instead of showing you something that is still incomplete."

R13 (Project Manager): "I will follow ASD or Kanban as Kanban flow would help us in mentoring the tasks."

\section{B. Development Team Proficiency}

Successful Agile methods can be achieved through team proficiency. For instance, team communication skills are very important when selecting Agile methods. Such proficiency may differ from one firm to the next, so this factor has to be considered when selecting a specific Agile method. It is crucial for project managers to confirm the proficiency of the team and on this basis, they can follow-up on what to do and which Agile methods to select. The description of the factors that have been highlighted under the category of team proficiency are provided in this sub-section.

1) Team communication skills: The success of adopting Agile methods requires extensive communication skills. The analysis revealed that the team communications skills affect the selection of Agile methods. If the team is co-located and their communication skills are poor, then there is a need for improvement. Respondents contended that XP and SCRUM are very suitable to improve the communication skills among the team:

R23 (Agile Coach): "I would try to have an assessment of problem solving among members, how to go about their work, how they communicate and collaborate among themselves and try to suggest different ways that help them to learn, and in this regard, SCRUM and XP practices will build communication. Pair programming, retrospectives are examples of how to help your team communicate."

R4 (Chief Executive Officer): "SCRUM forces us to communicate in different stages of the project."

However, if the team members are distributed or if there are solo developers, then the communication between them will be less. In this case tailored Agile methods such as DAD is needed. Moreover, DevOps and Kanban methods are suitable when there is distribution:

R16 (Senior Consultant Partner): "You have to tailor your approach to reflect geographical distribution which affect the communication between developers, the way you communicate within the team, whether the team changed, and you have to get in people around and more often. You probably have more sophisticated planning and documentation. So, the way you 
work in that environment is different than if you work in a colocated environment but you can still be Agile by planning a bit in advance, this is how DAD works."

R18 (Chief Information Officer): "Kanban might have the steps of where you can conceive a story and someone picks up that story, there is nothing in parallel, it is all in sequence. So, we can control the work in progress (WIP). So, that is different approach for the same problem and it will be conceivable that it is easier to support a developer in one location and QA in another location in different time zones, if at least your developer finishes the work and then called up the QAs in different time zone "Hey I have just finished story A, B and C", can you comment testing. Then possible answer of the QAs to the PO at the end of the day would be, "Hey I just finish testing these, check them for acceptance". It is conceivable that Kanban may work better in this progress rather than say SCRUM. So, Kanban is better for a distributed team.”

R23 (Agile Coach): "In DevOps, Solo developer builds, then deploys to package the functions, which have dependencies and then transfers them to the server. So, it really helps those solo developers to communicate with others without even knowing that but normally solo developers are really experts in continuous integration and delivery."

2) Team domain knowledge: Excellent team domain knowledge has been found to contribute to the success of Agile methods' use [13]. Team's domain knowledge is considered as an important factor in the selection of Agile methods [14]. The analysis of this empirical study revealed that team's domain knowledge influence the selection of Agile methods, if the team does not really understand the project to be built and how it will match the customers' need, then Lean Startup would be the optimal method to be selected:

R17 (Senior Agile Developer): "We have implemented a new project for the deaf and we did not know the details or the information about the project as it was not clearly defined ... we found that Lean Startup to be the most appropriate method because it allowed us to examine the output, it helped us to organize things in a good manner."

In addition, it was found that DAD, XP and DSDM would be appropriate when the domain knowledge is low:

R5 (Project Manager): "DAD, Lean Startup and DSDM emphasize on understanding the customers well before you start the projects, especially when the team does not understand the customers' needs."

R19 (Agile Trainer \& Consultant): "If there is no domain knowledge, I will focus more on applying pair programming and I will bring the people who requested the business to be available with us all the time. So, XP practices are very important in this case."

However, if the team domain knowledge is high or they have a good idea of the product backlog, then the team could move toward Lean development, Kanban and DevOps.

R3 (Delivery Manager): "If the team is consistent and knows the domain properly, indicating good domain knowledge, I will go toward Lean Development or Kanban, then we can just focus on improving the flow."

R1 Project Manager: "The knowledge of domain for the developers must be very high when selecting DevOps."

3) Team competency: Competency in this empirical study refers to team maturity (whether a team has real-world experience in using a specific Agile method or has built similar systems in the past using a specific Agile method) and the technical skills of the team to be able to adopt a specific Agile method. Both factors are discussed as follows:

a) Team Maturity: Different teams will need different things at different points of time, based on their maturity in using Agile methods. Therefore, selecting the method can be aligned with what the development team are familiar with and how matured are they in using Agile methods. So, most of the organizations select an Agile methods including many practices based on the team maturity. If the team in new to Agile methods, then the most appropriate Agile methods are the prescriptive methods and SCRUM is an optimum example of that:

R15 (Director of Development Management): "SCRUM is suitable for this group since they are beginners in using Agile methods."

In addition, it was found that DSDM is also appropriate for new Agile adopters since it is prescriptive as well. Additionally, Lean Startup can be used when the team is not matured in using Agile methods:

R6 (Agile Consultant): "We consider the team capability when selecting a specific Agile method. Some of the teams are not familiar with Agile methods. So, if the team capability is low, then we will give them some leverage on how to use Agile method by using either SCRUM, DSDM or Lean Startup."

However, when the team is mature in using prescriptive Agile methods, they should start thinking of adopting advanced Agile methods such as DevOps, Kanban, Lean Development, FDD. The latter methods require the team to be mature and have high competency in using Agile methods:

R1 (Project Manager): "If the team is not matured, then DevOps is not a choice."

R23 (Agile Coach): "Kanban is a cancer when the team is not an expert in using Agile methods, so we go back to SCRUM."

R6 (Agile Consultant): "If the team has high capability, they are competent and experienced in using Agile methods. Then we can choose Lean development or FDD."

b) Team technical skills: Technical skills of the team are an important factor, which affect the selection of Agile methods. In many organizations low skilled people exist who cannot use specific methods or they lack the technical skills, which allow them to adopt specific methods. Methods such as TDD and XP require high technical skills in order to be selected since most of their practices are engineering practices: 
R20 (Team Leader and Development Manager): "The technical skills should be high when adopting XP, as new programmers will never be able to use TDD as they will not think of testing before coding. Juniors will always face difficulties when adopting TDD."

In addition, it was found that DevOps require the team to have high technical skills to be able integrate, deliver automatically, test, monitor and deploy small chunks of software together.

R17 (Senior Agile Developer): "To adopt DevOps, the technical skills should be very good especially when considering the tools that should be used by the team. Many developers do not know how to use the DevOps, particularly when it comes to automation of the software delivery."

Adding together, Agile modeling and FDD require the team to be technically skilled, especially the modeling and the architecture skills.

R5 (Director of the Agile Organisation): "The technical architecture skills are highly needed when Agile modeling or FDD is selected."

\section{Customer Involvement}

It is important for customers to be actively involved in Agile methods procedure as without this involvement, then the team should opt for the Agile methods that are appropriate in understanding the customer's needs in advance. For instance, Adaptive Software Development (ASD) method emphasized on using joint application development session to increase customer involvement [15]. Hence, the customer should be encouraged and convinced of the significance of his involvement. Under this category, customer collaboration, customer domain knowledge and customer commitment are the main factors affecting the selection of Agile methods.

1) Customer collaboration: Customer collaboration in traditional methods such as waterfall is achieved by providing the requirements in the beginning and feedback towards the end, generally there is no regular interactions and collaborations between the customer and the development team. However, this is not the case when using Agile methods where the customer should interact and collaborate well with the team. Fortunately, with the increased number of Agile methods low and high customer collaboration can be handled. For instance, the analysis of this empirical study revealed that XP, SCRUM and Kanban will never be suitable when the customer is not collaborating well with the development team:

R15 (Director of Development Management): "If there is no customer collaboration, your project will fail for sure. SCRUM without customer collaboration will fail. Kanban is the same."

R12 (Principal Consultant): "XP practices might not help at all when the customer is not collaborating. Even, on-site customer will not help if the customer is not collaborating."

However, other Agile methods are appropriate even when customer is not collaborating well and this includes Adaptive Software Development (ASD), Agile modeling and Lean
Startup. Both methods assist in understanding the customers well and being able to find the appropriate way to get them involved and/or understand their requirements in advance.

R16 (Senior Consulting Partner): "Agile modeling techniques and regular demos would help in understanding the customer and helping them to collaborate well. So, these are the main practices that would help when the customer is not collaborating as expected."

R19 (Agile Trainer \& Consultant): "If the customer is not collaborative or not committed, then either the project will fail or I need to follow Lean Startup to be able to understand their needs effectively."

R12 (Principal Consultant): "Adaptive software development force the customer to get involved by developing simple prototypes during regular JAD sessions."

In addition, scaled Agile methods such as, DAD and SAFe are also appropriate when the customer is not collaborative as expected. For instance, DAD and SAFe encourage the team to understand the customer needs in advance.

R7 (Project Manager): "Many customers are not free or are careless to check each sprint; they just agree on what we provide for them, but at the end of the project we will get a lot of complains. In this case, we should use DAD or SAFe, both help in envisioning the needs of the customer in advance."

2) Customer domain knowledge: The customer or customer representative should have the ability to regularly provide the project requirements when using Agile methods. The analysis on or empirical study revealed that customer knowledge of their needs is an important factor, which affects the selection of Agile methods. Lean Startup, DAD, SAFe and FDD methods were found to be helpful when the customer domain knowledge is low. For instance, SAFe emphasizes on using UX when the customer domain knowledge is low:

R12 (Principal Consultant): "UX might be the best when the customer domain knowledge is low because this gets them into testing stage. You are really looking at it from the customer experience point of view. FDD might be also appropriate if you are able to select different customers looking for different things."

R16 (Senior Consulting Partner): "DAD is appropriate when there is a need for initial planning to solve dependencies issue and initial requirements gathering, especially when the customer does not know what he exactly needs. This calls for more architecture for the same issue including the team's understanding of what exactly to implement. Lean Startup is also appropriate"

R5 (Project Manager): "Also UX is another discipline technique. So, they do customer development, customer interview, prototyping and basically it fits into Agile. SAFe emphasizes on adopting UX. It complements Agile of the incremental development of product or service and through demos, rapid and frequent feedback from the customer can be obtained for better understanding and definition of the problem." 
Other methods such as XP and SCRUM, require the customer to have high domain knowledge to be active during the development:

R15 (Director of Development Management): "If the customers have high knowledge of what they want, they understand what they need exactly, then I will use XP method."

R2 (VP of Engineering and Operation): "If the customer knowledge of domain is high, then SCRUM can be the most suitable one."

3) Customer commitment: Customer commitment refers to the customer's active, motivated and responsible character in providing adequate requirements and testing the delivered sprints. However, the analysis of our empirical data revealed that customer commitment may differ from one project to another, as some might be busy and they might not be able to be involved as expected during the development processes. Based on the interview it was found that DAD method could be appropriate when the customer commitment is low as DAD assists in defining roles and responsibilities for the customer that can be structured forward. Kanban and SCRUM will not work well and there is a need to apply a bit of up-front planning at the planning stage:

R19 (Agile Trainer \& Consultant): "When there is no customer commitment, Kanban and SCRUM will not work well, and you need to apply a bit of up-front planning at the planning stage. You could follow DAD guidance in this case"

It was also found that Lean Startup is appropriate when the customer commitment is low. Lean Startup allows Agile team to understand the requirements and the needs in advance:

R19 (Agile Trainer \& Consultant): "If the customer is not collaborative or not committed, then either the project will fail or you need to follow Lean Startup to be able to understand their needs properly."

SCRUM method will be suitable when the customers are highly committed, especially in following the sprint planning. For instance, if it is two weeks sprint, then the customer should be committed to verify the work every two weeks.

R21 (Project Manager): "Having SCRUM is a bad idea when the customer is not committed because staff has to be sent out and this particular sprint will deliver multiple times (1, 2 , 3) without feedback. Then you'll move on to 4, 5, 6 assuming that everything is going to be okay, but then you get stuck. It really depends on the nature of how committed your clients/stakeholders are when they get involved."

\section{Organizational Culture (Type of Organization)}

Generally, Agile methods assume the team members are working in a collaborative manner rather than in a hierarchical one. Nevertheless, this empirical analysis revealed that some organizations have a hierarchical structure but are eager to be Agile. Therefore, in order to use Agile methods, the organization has to adapt its culture, and for this, Kanban and SAFe methods could lend a hand as they allow top management to monitor the progress of the development:
R10 (Senior Consultant): "Do you know an organization which is extremely hierarchical but still really agile? It is called TOYOTA. So, Kanban and Lean development methods could be adopted when there is a need to control the process."

R12 (Principal Consultant): "If you are truly hierarchical, then I think you have to go with something that is well planned ahead. Because the hierarchy always demands what the team is going to build and when it is going to be ready. They might say "I need this now" or "I need this commitment" and thus, things like Kanban and SAFe can work as they have predictable sketch along the way."

In addition, it was also found that SCRUM method would help when the organization is at the transition stage "immature team" but the development team members themselves should be collaborative.

R6 (Agile Consultant): "Even with hierarchical culture, we can choose SCRUM method if the team members themselves collaborate with each other."

R9 (Change Leader): "If the organization is already agile, I would opt for Kanban rather than SCRUM, particularly when the culture is hierarchical." Agile Adoption

Finally, DSDM would also assist the management to control the work.

R8 (Director of the Agile Organization): "I do not think DSDM improve the quality when compared to TDD and XP but for some organizations, which are highly bureaucratic, DSDM might be helpful."

\section{E. Project Constraints}

The development team can employ any appropriate method to their preferences, but with strict constraints, depending on the context. For instance, some organizations are faced with the challenge of dealing with quality products, while others have issues with time to market, scope variability and project costs. In addition, the analysis of the empirical result revealed that requirement volatility and cost of change are other challenges that organizations face. These are the primary constraints that have to be kept under consideration during Agile methods selection. In other words, the team has to determine the most suitable method that is consistent with the constraints. The project constraints are described in detail on the basis of the feedback obtained from the respondents in the next paragraphs by category.

1) Time: Different organizations are faced with different challenges, with one of the main challenges being time to market, which affects the selection of Agile methods in two ways. First of all, the speed of the delivery and in this regard, DevOps was found to focus on this purpose:

R1 (Project Manager): "We want to have quick and fast delivery, so we adopt DevOps."

In addition, it was also found that XP and Kanban also focus on delivering the project fast. XP focuses on coding and continuous integration and Kanban focuses on managing the flows instead of managing the iterations, which makes the progress faster: 
R15 (Director of Development Management): "If the team has good technical skills and experience in using XP practices, they could use Kanban to manage the work, which will make the process faster, especially if the customer provides regular and daily feedback."

R17 (Senior Agile Developer): "we will immediately use XP to develop the project fast especially if we have a team who has good programming skills, loves coding and has skills of continuous integration."

Sometimes, the customer might request a fixed schedule for each sprint of the project so the sprints delivery should be at a specific date, then the methods are called for which enables this and they are SCRUM and DAD:

R11 (Product and Management Consultant): "With timebound scope, I will go with SCRUM, otherwise I will go with Kanban."

R12 (Principal Consultant): "If you got really strong contractual requirements, you may have something that needs delivery of functions at each step of the way or penalty payment, in which case you need to have something that is more disciplined in order to be able to manage it more closely. In this case, DAD would be a very good choice."

2) Quality: Agile methods in general, focus on increasing the quality of the products. Some Agile methods and Agile methods' practices are required when the challenges are to deal with quality of products to effectively and efficiently accelerate activities [16, 17]. XP methods and TDD were found to be the most appropriate methods to increase the quality and deal with such challenges:

R8 (Director of the Agile Organization): "In almost all cases, to get high quality product I'd recommend *also* to adopt the XP practices."

R20 (Team Leader and Development Manager): "The adoption of TDD would help a lot in improving the quality."

In addition to XP and TDD, it was also found that DAD and SCRUM retrospective increase the quality. DAD contains a bit of architecture visioning and upfront planning and SCRUM retrospective helps the team to solve the quality issues:

R16 (Senior Consulting Partner): "Also, I might opt for upfront architecture envisioning for better quality and in this case we could use DAD or Agile modeling."

R5 (Project Manager): "SCRUM Retrospective improves the quality, especially when the team focuses on the items that should be delivered with a hundred percent quality."

3) Scope variability: The scope of an Agile project is not defined by detailed requirements but by the high level requirements such as, user stories. Based on the analysis, it was found that scope variability affects the selection of Agile methods in that if the scope variability is high and there are difficulties in batching the requirements into two weeks' time, in which case, Kanban and Lean development are the appropriate methods to be used:
R10 (Senior Consultant): "The more the scope is unpredictable, the less lead time it can endure. Kaizen's premise is fail fast, find problems and fix them and both apply Kanban and Lean in this case, which will decrease the cost of implementation and help the team to limit the work in process and focus on one thing during the implementation."

Moreover, SCRUM method will not be suitable when the scope variability is high, especially if the predictability of the scope is not stable:

R11 (Product and Management Consultant): "Understanding the context and the problem calls for scope predictability in a stable environment, then SCRUM would be a good choice. This can be done in 2-4 weeks' time. You need to deliver and freeze, then employ SCRUM but if scope predictability is a dynamic environment, then go with Kanban"

4) Requirements variability: Requirements volatility means that your requirements are changing dramatically on daily basis or sometimes on hourly basis. So, if the requirements volatility is high, then Kanban is the most appropriate method to be selected. Therefore, it depends on whether the requirements of the customer are stable or not:

R14 (Chief Knowledge Engineer)"If you are working with highly volatile requirement, then you need an Agile method, which can help you deal with that - in other words, you need iterative development, very short iterations, user stories and elaborated just in time. So, Kanban is suitable for that."

R21 (Project Manager): "Kanban could be very good if the management of the banks keep adding new requirements in the middle of each sprint."

In addition, DAD could be used to have a clear picture of the final product in advance, so if there is possibility of requirement changes, it will be clear before starting the implementation of the product. Also Lean methods could be used to allow a failing team to get early feedback:

R7 (Project Manager): "If the scope is not clear but the requirements are stable and can be batched, then we can adopt SCRUM but if the requirements are not stable and there can be daily changes, then something, which allows upfront planning to understand the requirements can be adopted, in which case, DAD can guide on how to understand the big picture of the project in advance."

SCRUM is suitable when the requirements are stable or at least the changes are not on a daily basis. So, it can be used when the requirements keep on changing but if the requirements volatility is high, then it's better to use Kanban:

R6 (Agile Consultant): "SCRUM can be used for brand new projects but if the requirements are volatile and changing rapidly then it's better not to use SCRUM for brand new projects."

5) Cost/Value/ROI: In general, most Agile methods focus on reducing the cost of software development. However, there are some methods which emphasize on being cost-effective. So, by adopting that specific Agile method, the development cost will be decreased. This can be achieved by decreasing 
any extra features, delays and defects, which decreases the cost of the implementation. Some methods help in providing what is being asked by the customer or limiting the work to deliver the right requirements only without providing extra things, which might not provide value for the customer. DevOps/Continuous delivery and XP methods were found appropriate in decreasing the project cost as supported by different Agilists as follows:

R14 (Chief Knowledge Engineer): "I personally take and advice my team to adopt is the XP philosophy. Anything below that just results in a technical debt and long term cost."

R17 (Senior Agile Developer): "For projects, which have small budgets, the best method to be used is DevOps as it helps in delivering the project faster."

In addition, Kanban, Lean Startup and Lean development were found to be appropriate in decreasing the cost. They focus on developing the right requirements, which return investment in a timely manner. In addition, they focus on eliminating waste by not performing unnecessary things. For instance, Kanban helps the team to focus on small pieces of work to be able to control the flow, so unnecessary ceremonies will be prevented:

R8 (Director of the Agile Organization): "When there is a cost constraint, I might consider having a preference for Lean or Kanban type approach because it allows us to focus on eliminating waste."

\section{DISCUSSION}

Based on the results above, Agile methods selection model in Fig. 1 illustrates the factors that have been found to affect the selection of an Agile methods. The Agile methods, whose efficacy is affected by these factors are shown in the center box in Fig. 1. Each factor comprises different values that can be used in the selection of different Agile methods.

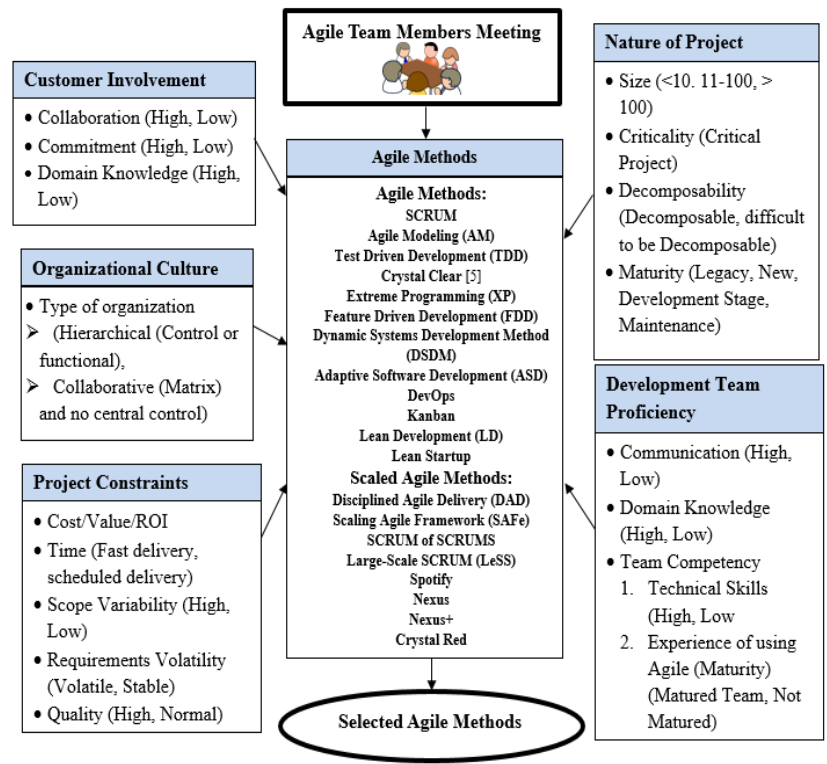

Fig. 1. Agile Methods Selection Model.
The analysis grouped the factors into five categories. By considering the degree to which each of the identified factors is important to the project, Agile team members should conduct a meeting to discuss the targeted projects to be implemented and the factors, which affect the selection of the methods. Team meeting in the selection of Agile methods was supported by different respondents to select the appropriate Agile methods. The findings would contribute in the selection of the appropriate Agile methods for different development projects in different organizations, and this leads to the delivery of high quality software with reasonable cost and efficient time usage.

\section{CONCLUSION}

Agile methods selection should be considered at each and every organization while keeping different teams and projects under consideration. Selecting the right Agile methods has a pertinent influence on the success of implementing any project. This research presented various recommendations about the situations in which an Agile methods can be suitable. Individual interviews were used to explore the different practices that Agile teams normally follow to select the appropriate Agile methods for different projects and/or different teams at different organizations. The results from the data collection were imported into NVivo, a qualitative data analysis software package. Several factors were identified including how they could be used in selecting Agile methods based on grounded theory analysis. The result indicates how each factor could be used in the selection and this is the key to answering the main research question of this study. Further work can be undertaken to investigate the appropriate Agile methods and Agile methods practices for requirements reuse, which is an important topic. In addition, other factors and new Agile methods may emerge for the development of specific projects [18]. So, selecting the appropriate Agile methods can be investigated again.

\section{ACKNOWLEDGMENT}

We acknowledge the Faculty of Information Science and Technology at Universiti Kebangsaan Malaysia (UKM) for supporting this research under Fundamental Research Grant Scheme project (FRGS/2/2013/ICT01/UKM/02/2).

\section{REFERENCES}

[1] S. C. Misra, V. Kumar, and U. Kumar, "Identifying some important success factors in adopting agile software development practices," J. Syst. Softw., vol. 82, no. 11, 2009, pp. 1869-1890.

[2] M. K. Alqudah and R. Razali, "Key Factors for Selecting an Agile Method: A Systematic Literature Review," Int. J. Adv. Sci. Eng. Inf. Technol., vol. 7, no. 2, 2017, pp. 526-537.

[3] A. Sharma and R. K. Bawa, "A Framework for Agile Development Method Selection using Modified PROMETHEE with Analytic Hierarchy Process," Int. J. Comput. Sci. Inf. Secur., vol. 14, no. 8, 2016, p. 846.

[4] A. Alliance, "Agile manifesto," Online at http://agilemanifesto.org/, vol. 6, no. 1, 2001.

[5] A. Cockburn, Crystal Clear: A Human-Powered Methodology for Small Teams: A Human-Powered Methodology for Small Teams. Pearson Education, 2004.

[6] D. J. Anderson, “Kanban.” Blue Hole Press Sequim, WA, 2010.

[7] J. Sutherland and K. Schwaber, "The scrum guide. The definitive guide to scrum: The rules of the game," Scrum. orgOctober, 2013. 
[8] K. Beck, Extreme programming explained: embrace change. addisonwesley professional, 2000.

[9] B. Sayed, Z. Shamsi, and M. Sadiq, "A Method for the Selection of Agile Methods Using AHP," in Proceedings of the 5th International Conference on Frontiers in Intelligent Computing: Theory and Applications, 2017, pp. 297-303.

[10] H. Ayed, B. Vanderose, and N. Habra, "A Context-Driven Approach for Guiding Agile Adoption: The AMQuICk Framework," ICSEA 2015 Tenth Int. Conf. Softw. Eng. Adv. A, no. c, 2015, p. 6,

[11] B. G. Glaser, "The grounded theory perspective III: Theoretical coding". Sociology Press, 2005.

[12] A. Strauss and J. Corbin, "Basics of qualitative research: Procedures and techniques for developing grounded theory." Thousand Oaks, CA: Sage, 1998.

[13] M. R. Hilkka, T. Tuure, and M. Rossi, "Is extreme programming just old wine in new bottles: A comparison of two cases," J. Database Manag., vol. 16, no. 4, p. 41, 2005.
[14] F. Q. B. da Silva, A. C. C. França, M. Suassuna, L. M. de Sousa Mariz, I. Rossiley, R. C. de Miranda, T. B. Gouveia, C. V. Monteiro, E. Lucena, E. S. Cardozo, and E. Espindola, "Team building criteria in software projects: A mix-method replicated study". Information and Software Technology, vol. 55, no. 7, 2013, pp. 1316-1340.

[15] F. Paetsch, A. Eberlein, and F. Maurer, "Requirements engineering and agile software development," in Enabling Technologies: Infrastructure for Collaborative Enterprises, 2003. WET ICE 2003. Proceedings. Twelfth IEEE International Workshops on, 2003, pp. 308-313.

[16] M. Alqudah and R. Razali, "A review of scaling agile methods in large software development,” Int. J. Adv. Sci. Eng. Inf. Technol., vol. 6, no. 6, 2016.

[17] M. Alqudah and R. Razali, "A comparison of scrum and Kanban for identifying their selection factors," in Electrical Engineering and Informatics (ICEEI), 6th International Conference on, 2017, pp. 1-6.

[18] K. Almajed, W. Medhat and T. El-Shishtawy, "An Iterative Hybrid Agile Methodology for Developing Archiving Systems". International Journal of Software Engineering \& Applications (IJSEA), vol. 10 no. 12019. 